\title{
Identification of recurrent mutational events in anorectal melanoma
}

\author{
Hui Min Yang ${ }^{1,2,6}$, Susan J Hsiao ${ }^{1,6}$, David F Schaeffer ${ }^{2}$, Chi Lai ${ }^{3}$, Helen E Remotti ${ }^{1}$, \\ David Horst ${ }^{4}$, Mahesh M Mansukhani ${ }^{1}$ and Basil A Horst ${ }^{1,5}$ \\ ${ }^{1}$ Department of Pathology \& Cell Biology, Columbia University Medical Center, New York, NY, USA; \\ ${ }^{2}$ Department of Pathology \& Laboratory Medicine, University of British Columbia, Vancouver, BC, Canada; \\ ${ }^{3}$ Department of Pathology and Laboratory Medicine, University of Ottawa, Ottawa, ON, Canada; \\ ${ }^{4}$ Pathologisches Institut, Ludwig-Maximilians-Universitaet, Muenchen, Germany and ${ }^{5}$ Department of \\ Dermatology, Columbia University Medical Center, New York, NY, USA
}

\begin{abstract}
Anorectal melanoma is a rare disease that carries a poor prognosis. To date, limited genetic analyses confirmed KIT mutations as a recurrent genetic event similar to other mucosal melanomas, occurring in up to $30 \%$ of anorectal melanomas. Importantly, a subset of tumors harboring activating KIT mutations have been found to respond to c-Kit inhibitor-based therapy, with improved patient survival at advanced tumor stages. We performed comprehensive targeted exon sequencing analysis of 467 cancer-related genes in a larger series of 15 anorectal melanomas, focusing on potentially actionable variants based on gain- and loss-of-function mutations. We report the identification of oncogenic driver events in the majority (93\%) of anorectal melanomas. These included variants in canonical MAPK pathway effectors rarely observed in cutaneous melanomas (including an HRAS mutation, as well as a BRAF mutation resulting in duplication of threonine 599), and recurrent mutations in the tumor suppressor NF1 in $20 \%$ of cases, which represented the second-most frequently mutated gene after $K I T$ in our series. Furthermore, we identify SF3B1 mutations as a recurrent genetic event in mucosal melanomas. Our findings provide an insight into the genetic diversity of anorectal melanomas, and suggest significant potential for alternative targeted therapeutics in addition to c-Kit inhibitors for this melanoma subtype.
\end{abstract}

Modern Pathology (2017) 30, 286-296; doi:10.1038/modpathol.2016.179; published online 14 October 2016

Anorectal melanoma is a rare and highly lethal malignant neoplasm, comprising $\sim 1 \%$ of all melanomas and $<2 \%$ of anal tumors. ${ }^{1-3}$ The annual incidence in the United States is $\sim 0.3$ per million with a male to female ratio of $2: 3$, whereas other large population-based studies report higher incidence rates of 1.0 per million. ${ }^{1,4}$ The anal canal is best defined in vivo, extending from the rectal pubalis sling to the anal verge. As such, the anal canal has three epithelial zones: rectal/colonic mucosal zone, the transitional zone, and the squamous mucosal zone. The anal transitional zone varies in length and is comprised of variable mucosa. Although anorectal melanoma was historically thought to arise from anal

Correspondence: Dr BA Horst, MD, Department of Dermatology, Pathology \& Cell Biology, Columbia University Medical Center, New York Presbyterian Hospital, 630 West 168th Street, VC15207, New York 10032, NY, USA.

E-mail: bh2179@columbia.edu

${ }^{6}$ These authors contributed equally to this work.

Received 1 July 2016; revised 27 August 2016; accepted 30 August 2016; published online 14 October 2016 squamous epithelium, it was recognized that melanocytes are present within the anal transitional zone, as well as above the dentate line in the proximal anal canal/distal rectum within colorectal mucosa. ${ }^{5-7}$ Approximately $60 \%$ of melanomas are diagnosed in the anal canal and up to $40 \%$ in the rectum. ${ }^{1}$ Of note, anorectal melanoma in the United States shows a rising incidence. ${ }^{1}$

A unifying staging system for mucosal melanoma, including anorectal melanoma, is currently lacking, partially owing to rarity of the disease. A simplified three-tiered system for melanomas arising on the head and neck ${ }^{8}$ categorizes disease extent into clinically localized (Stage I), regional lymph node involvement (Stage II), and distant metastasis (Stage III), and was shown to correlate with outcome in a recent large retrospective series of anorectal melanomas. ${ }^{9}$ Surgical treatment appears effective for localized disease. ${ }^{2,10}$ However, overall survival for patients suffering from anorectal melanoma remains dismal, with 5 -year survival rates for Stage I, Stage II, and Stage III disease of $26 \%, 9.8 \%$, and $0 \%$, respectively. ${ }^{9}$ 
To date, analyses of few genetic loci in anorectal melanoma revealed that, similar to melanomas at other mucosal sites, mutations in BRAF and NRAS are significantly less frequent as compared with cutaneous melanomas, whereas activating KIT mutations represent a recurrent mutational event. ${ }^{11,12}$ One of the largest studies comprising 31 primary anorectal melanomas reported KIT mutations in $>30 \%$ of cases. $^{13}$ Importantly, whereas radical surgery and radiotherapy failed to improve survival, ${ }^{2,14}$ several prospective trials demonstrated clinical benefit of imatinib in patients with metastatic mucosal melanomas harboring activating KIT mutations, with response rates between 16 and 25\% and some responses lasting for longer than one year. ${ }^{15-17}$ However, secondary resistance eventually develops, and although alternative kinase inhibitors such as nilotinib have shown limited efficacy in c-Kit inhibitor-refractory disease, the overall prognosis for these patients remains poor. ${ }^{18}$ Furthermore, a majority of mucosal melanomas lack KIT mutations. Exploration of additional actionable mutational events is therefore crucial to refine molecular therapy for this subset of tumors, and to expand treatment options with the potential of improving survival in this devastating disease.

The development of targeted therapies in cancer has accelerated the development of molecular diagnosis, with the emergence of next-generation sequencing technologies as useful new tools in oncology and personalized medicine. In light of the limited data that includes mutation status of select oncogenes and tumor suppressors, ${ }^{11,13,19-21}$ we performed expanded molecular profiling of a larger series of anorectal melanomas.

We report the identification of annotated oncogenic driver events in the majority of anorectal melanomas (14 of 15 cases), with potential implications for targeted therapy.

\section{Materials and methods}

\section{Case Selection}

Fifteen cases of anorectal melanoma diagnosed between 1 January 1990 and 1 January 2015 with sufficient residual material for analysis were retrieved from the surgical pathology archives of the Columbia University Medical Center, New York, NY the Vancouver General Hospital, Vancouver, BC, and the Ludwig Maximillian University Tumor bank, Munich, Germany, with approval of respective Institutional Review Boards. Original diagnosis was based on clinical (anatomic site) and histologic features, and melanocytic lineage of tumors confirmed by immunohistochemistry. Clinical data were reviewed to confirm the absence of a prior history of melanoma. H\&E-stained sections and immunohistochemical stains of all study cases were reviewed to verify anatomic site, relationship to the anal transitional zone, as well as for assessment of an intraepithelial/in situ component. Where available, follow-up information was obtained from review of medical records. Survival time was defined as the time from initial diagnosis until last follow-up.

\section{DNA Extraction, Targeted Sequencing, and Data Analysis}

To enrich for lesional tissue, representative tumor areas were manually microdissected from formalin fixed, paraffin-embedded tissue sections. DNA was extracted using QIAcube (Qiagen, Hilden, Germany), according to the manufacturer's instructions. DNA was analyzed by the Columbia Molecular Pathology Combined Cancer Panel. ${ }^{22}$ In total, 200 ng of DNA was sheared to a median length of $200 \mathrm{bp}$ using a Covaris S2 Sonication system (Covaris, Woburn, MA, USA), and exonic sequence from 467 cancer-related genes was captured using custom Agilent SureSelect reagents (Santa Clara, CA, USA). Sequencing was performed on the Illumina HiSeq2500 (San Diego, CA) using Illumina TruSeq v3 chemistry and as 100bp paired-end reads (up to nine indexed samples per run). Demultiplexing was performed with CASAVA and alignment and variant calling was performed using NextGENe software (Softgenetics, State College, PA, USA), with the following parameters: 0 allowable ambiguous alignments, at least $90 \%$ of reads matching the reference genome, at least $10 \%$ variant allelic fraction and at least three variant reads required to call a variant. Single-nucleotide variants as well as small insertions and deletions were annotated and filtered by an in-house developed pipeline and evaluated by a molecular pathologist. In brief, variants were cross-referenced with the 1000 Genomes Project, OMIM, dbSNP and the Exome Variant Server. Variants with $>1 \%$ allele frequency, common variants present in our departmental database of variants identified in prior constitutional exome analysis, non-pathogenic variants reported in dbSNP as well as low-quality calls were filtered out. Variants were annotated with dsSNP, ClinVar, HGMD, OMIM, and COSMIC databases, as well as by predicted protein effect (using in silico predictors Provean and SIFT). Potential variants were manually curated and classified by literature review to evaluate for pathogenic changes consistent with protein function.

\section{Immunohistochemistry}

Immunohistochemical analysis was performed using a Ventana automated slide stainer and Ventana ultraView universal DAB detection kit, as we described previously. ${ }^{23}$ The following pre-diluted antibodies (Ventana, Tucson, AZ, USA) were used: MLH1 (clone G168-15), MSH2 (clone FE11), PMS2 (clone MRQ-28) and MSH6 (clone 44). 
Table 1 Clinical and pathologic features of anorectal melanomas

\begin{tabular}{|c|c|c|c|c|c|c|c|c|c|}
\hline Case no. & Age, sex & $\begin{array}{l}\text { Relationship } \\
\text { to ATZ }\end{array}$ & $\begin{array}{l}\text { Thickness } \\
\text { (mm) }\end{array}$ & $M I S$ & $\begin{array}{l}\text { Mitotic } \\
\text { rate } / \mathrm{mm}^{2}\end{array}$ & Histology & Metastases & $\begin{array}{l}\text { Alive/dead, } \\
\text { durvival (m) }\end{array}$ & Stage* \\
\hline 1 & $73 \mathrm{M}$ & Above & 4.5 & ND & 10 & Epithelioid & Liver, spleen, LN & DOD, 21 & III \\
\hline 2 & $83 \mathrm{M}$ & At & 9.2 & yes & 8 & $\begin{array}{l}\text { Epithelioid/ } \\
\text { spindled }\end{array}$ & Lung & DOD, 13 & III \\
\hline 3 & $48 \mathrm{~F}$ & Below & 4.5 & yes & 5 & Epithelioid & Adrenal, lung & DOD, 12 & III \\
\hline 4 & $64 \mathrm{~F}$ & Below/At & 5.1 & yes & 7 & Epithelioid & $\mathrm{LN}$ & Dead, 18 & II \\
\hline 5 & $68 \mathrm{~F}$ & Below & $\geq 5$ & yes & 33 & Epithelioid & $\begin{array}{l}\text { Lung, liver, } \\
\text { spleen, LN }\end{array}$ & DOD, 7 & III \\
\hline 6 & $45 \mathrm{M}$ & Above & $\geq 2.5$ & ND & 4 & Epithelioid & Unknown & Alive, 1 & - \\
\hline 7 & $81 \mathrm{M}$ & At & 7 & yes & 38 & Epithelioid & Negative & Alive, 30 & I \\
\hline 8 & $83 \mathrm{M}$ & Above/At & 46 & yes & 16 & $\begin{array}{l}\text { Epithelioid/ } \\
\text { spindled }\end{array}$ & LN & DOD, 9 & II \\
\hline 9 & $79 \mathrm{~F}$ & Below & $\geq 3$ & yes & 3 & Epithelioid & $\begin{array}{l}\text { Liver, } \\
\text { peritoneum, LN }\end{array}$ & Unknown & III \\
\hline 10 & $65 \mathrm{~F}$ & Above & 14 & ND & 20 & $\begin{array}{l}\text { Epithelioid/ } \\
\text { spindled }\end{array}$ & Liver & DOD, 12 & III \\
\hline 11 & $57 \mathrm{~F}$ & Below & 2.5 & yes & 4 & Epithelioid & Liver, LN & DOD, 16 & III \\
\hline 12 & $73 \mathrm{~F}$ & At & 4.5 & yes & 16 & Epithelioid & Liver, LN & DOD, 5 & III \\
\hline 13 & $77 \mathrm{M}$ & Below & $\geq 2.2$ & yes & 19 & Epithelioid & Negative & Dead, 3 & I \\
\hline 14 & $74 \mathrm{M}$ & Below/At & 3.9 & yes & 3 & Epithelioid & Unknown & Dead, 5 & - \\
\hline 15 & $57 \mathrm{~F}$ & Below & $\geq 11$ & ND & 8 & Spindled & Brain, liver, LN & DOD, 8 & III \\
\hline
\end{tabular}

Abbreviations: M, male; F, female; ATZ, anal transitional zone; MIS, melanoma in situ; ND, not determined due to colonic mucosal localization and/or extensive ulceration; LN, lymph node; DOD, dead of disease; *Ballantyne staging system.

\section{Microsatellite Instability Testing}

Microsatellite instability (MSI) testing was performed using 1-2 ng of DNA extracted from formalin fixed, paraffin-embedded tissue using the Promega MSI Analysis System (Promega, Madison, WI, USA), according to the manufacturer's instructions. Analysis of PCR products was performed on an ABI PRISM 3100-Avant genetic analyzer (Thermo Fisher Scientific, Waltham, MA, USA).

\section{Results}

\section{Clinical and Histologic Characteristics}

The clinical and histologic findings of fifteen cases of primary anorectal melanoma are summarized in Table 1. Patient age ranged from 45 to 83 years, with a mean age of 68.5 years and median age of 73 years. Cases included seven men and eight women. In four cases, the tumor was centered above the anal transitional zone within colonic mucosa and in 11 cases at or below the anal transitional zone (transitional type mucosa/squamous mucosa). Melanoma in situ was identified in all tumors occurring at or below the anal transitional zone, except in one case which showed extensive ulceration. Careful review of clinical data revealed no history of melanoma at other sites in any of the patients. Clinical follow-up data was available for 14 patients and ranged from 1 to 30 months after initial diagnosis. Two patients were alive at last follow-up, with no documented evidence of metastasis (Table 1).

A representative case of invasive anorectal melanoma is depicted in Figure 1.

\section{Molecular Findings}

Next-generation sequencing analysis of exonic sequence from 467 cancer-related genes (Columbia Combined Cancer Panel) was successfully performed in all 15 cases of anorectal melanoma, with a mean depth-of-coverage of the region of interest of $731 \times$. On average, 14.1 non-synonymous or small insertion/deletion variants were detected per case (range 4-33 variants/case), the majority of which represented variants with unknown significance (Supplementary Table 1). Of note, driver mutations, defined as pathogenic alterations recurrent in human cancers and conferring a growth advantage, ${ }^{24}$ were identified in 14 of 15 cases $(93 \%)$ (Figure 2). Furthermore, a majority of these driver events represent actionable mutations, with significant potential to enhance targeted therapy for this melanoma subtype (Table 2). In most cases (11 of 15), a single driver mutation was identified, whereas three cases showed two and one case showed three driver mutations (Figure 2).

The most frequently mutated gene in our series of anorectal melanomas was KIT, with mutations identified in 5 of 15 tumors (33\%). Three mutations (W557R, V560D, V559A), previously reported as oncogenic, were found in exon 11 (juxtamembrane domain), and are expected to predict sensitivity to tyrosine kinase inhibitors such as imatinib, nilotinib, or sorafenib (Table 2). In addition, we identified two KIT mutations in exon 17 involving the distal tyrosine kinase domain (Y823D, D820Y), which are known to correlate with acquired imatinib resistance in gastrointestinal stromal tumors but 


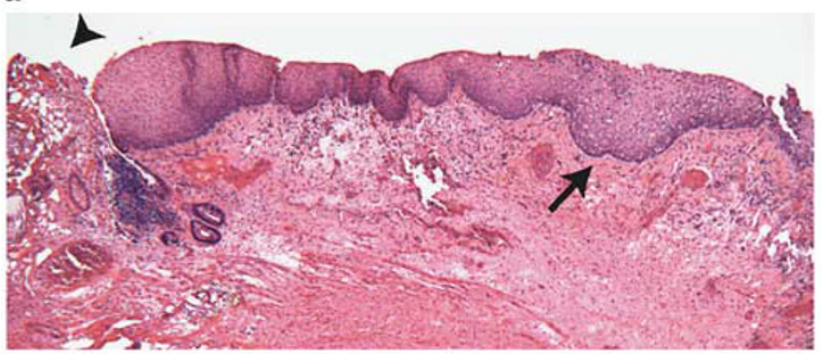

C

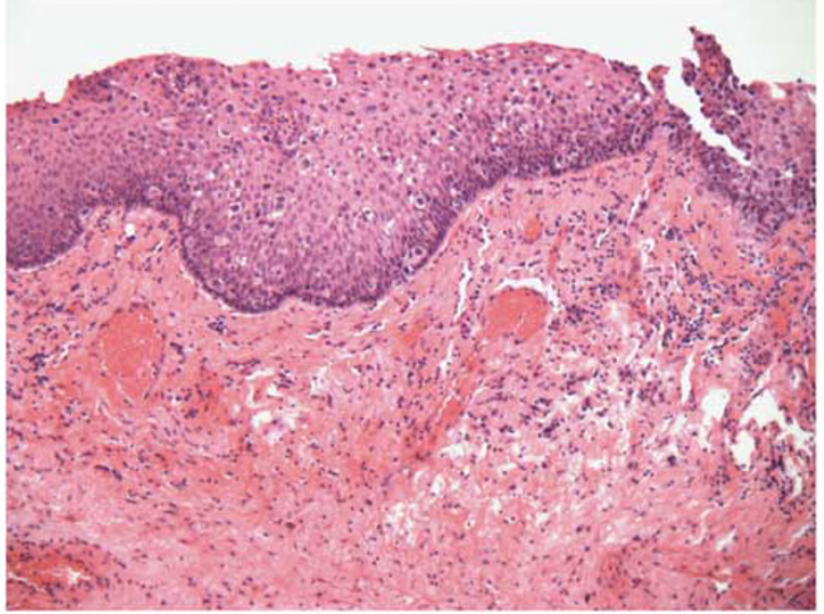

e

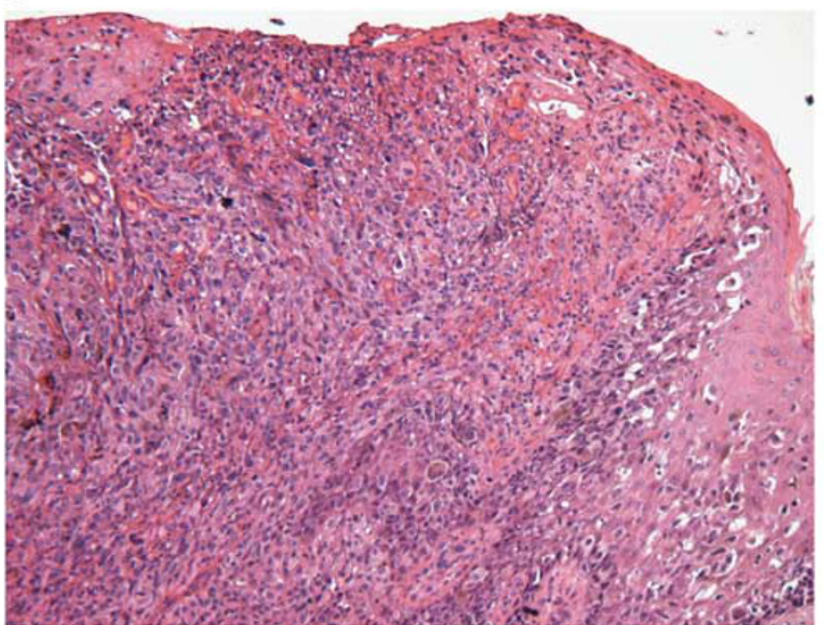

b

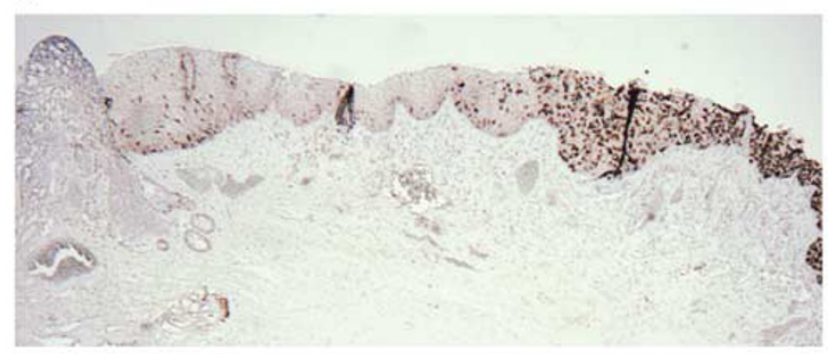

d

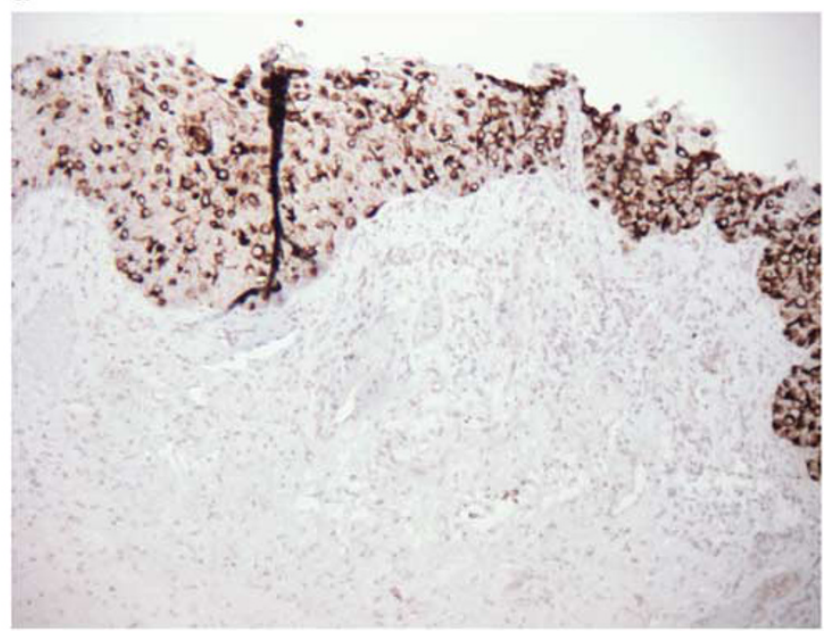

f

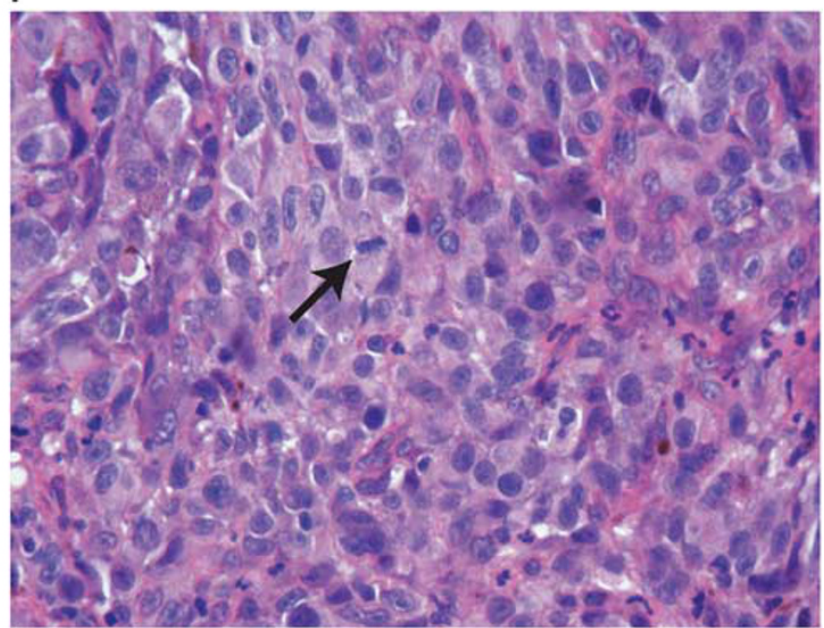

Figure 1 Representative case of anorectal melanoma, histologic features. (a) Atypical intraepithelial melanocytic proliferation within squamous mucosa of the anal transitional zone (arrow), adjacent colonic mucosa (arrowhead) (original magnification $\times 40)(b)$ Atypical intraepithelial melanocytes stain strongly positive for Melan-A (original magnification $\times 40$ ). (c, d) Melanoma in situ with prominent pagetoid scatter of atypical melanocytes (c), highlighted by Melan-A immunohistochemical stain (d) (original magnification $\times 100)$. (e) Invasive melanoma with nests and sheets of atypical melanocytes. Note overlying ulceration and pigment production (original magnification $\times 100$ ). (f) Invasive melanoma. Proliferation of atypical melanocytes with pleomorphic nuclei and mitotic activity (arrow) (original magnification $\times 400$ ).

described in one case of mucosal melanoma showing a partial treatment response, ${ }^{17}$ (Table 2). Overall, these results are in agreement with previous studies and confirm KIT mutations as a predominant mutational event in anorectal melanomas.
Anorectal Melanomas Harbor Recurrent Mutations in NF1, as well as Mutations in MAPK Pathway Effectors Distinct from Cutaneous Melanomas

Oncogenic mutations in genes affecting $R A S$ and its canonical downstream effectors were seen in 3 of 15 


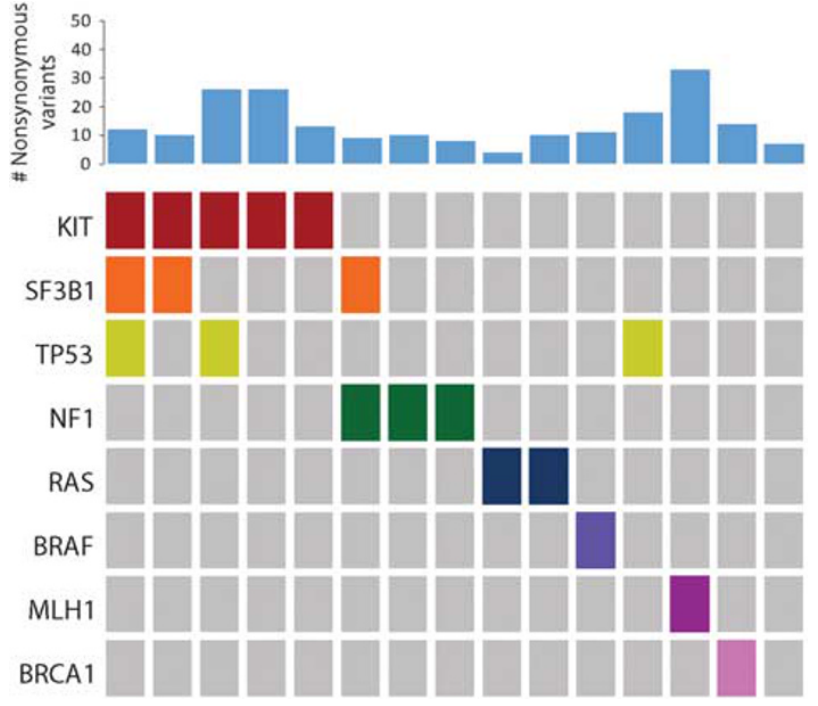

Figure 2 Overview of genetic alterations detected in anorectal melanoma. Number of non-synonymous and insertion/deletion variants detected per case (top panel). Pathogenic driver mutations, defined as recurrent oncogenic events reported in the COSMIC database, are indicated by colored boxes (lower panel).

Table 2 Driver mutations, affected pathways, and potential inhibitors

\begin{tabular}{|c|c|c|c|c|}
\hline $\begin{array}{l}\text { Case } \\
\text { no. }\end{array}$ & Gene & Mutation & $\begin{array}{l}\text { Affected } \\
\text { pathway }\end{array}$ & $\begin{array}{l}\text { Potential } \\
\text { inhibitors }\end{array}$ \\
\hline 1 & NF1 & p.K1844fs & MAPK/PI3K & MEKi \\
\hline 2 & KIT & Y823D & MAPK/PI3K & - \\
\hline 3 & $\begin{array}{l}\text { KIT } \\
\text { SF3B1 } \\
\text { TP53 }\end{array}$ & $\begin{array}{l}\text { p.V560D } \\
\text { p.R625C } \\
\text { p.W53X }\end{array}$ & MAPK/PI3K & RTKi \\
\hline 4 & $\begin{array}{l}\text { KIT } \\
\text { SF3B1 }\end{array}$ & $\begin{array}{l}\text { p.W557R } \\
\text { p.R625H }\end{array}$ & MAPK/PI3К & RTKi \\
\hline 5 & KIT & p.V599A & MAPK/PI3К & RTKi \\
\hline 6 & $\begin{array}{l}\text { KIT } \\
\text { TP53 }\end{array}$ & $\begin{array}{l}\text { p.D820Y } \\
\text { p.Y220C }\end{array}$ & MAPK/PI3K & RTKi \\
\hline 7 & MLH1 & p.G67R & DNA repair & - \\
\hline 8 & BRCA1 & p.T557fs & DNA repair & - \\
\hline 9 & - & - & - & - \\
\hline 10 & $\begin{array}{l}\text { NF1 } \\
\text { NF1 } \\
\text { SF3B1 }\end{array}$ & $\begin{array}{l}\text { p.H1251fs } \\
\text { p.Y2629X } \\
\text { p.R625C }\end{array}$ & MAPK/PI3K & MEKi \\
\hline 11 & $B R A F$ & p.T599dup & MAPK & MEKi \\
\hline 12 & $\begin{array}{l}\text { NF1 } \\
\text { NF1 }\end{array}$ & $\begin{array}{l}\text { p.W571X } \\
\text { c. } 204+1 G>C\end{array}$ & MAPK/PI3К & MEKi \\
\hline 13 & TP53 & p.C242Y & DNA repair & - \\
\hline 14 & HRAS & p.Q61R & MAPK/PI3K & MEKi \\
\hline 15 & NRAS & p.G12A & МАРК/РІ3К & MEKi \\
\hline
\end{tabular}

Abbreviations: Dup, duplication; fs, frameshift; MAPK, mitogenactivated protein kinase (Erk); MEKi, MAPK kinase inhibitor (eg, selumetinib, trametinib, binimetinib); ${ }^{38}$ RTKi, inhibitors with activity against receptor tyrosine kinases (eg, imatinib, nilotinib); bold, mutations newly discovered in mucosal melanomas. cases of anorectal melanoma (20\%, Table 2). Interestingly, in addition to one NRAS (G12A) mutation, we also identified one case each carrying an HRAS (Q61R) mutation, more typically seen in Spitz nevi, ${ }^{25}$ as well as a rare three-base-pair insertion resulting in duplication of threonine at codon 599 in the $B R A F$ activation loop (p.T599dup). This mutation was previously described to display in vitro kinase activity comparable to $B R A F$ (V600E), ${ }^{26}$ the predominant mutation in cutaneous melanomas. Significantly, we furthermore detected recurrent deleterious mutations in $N F 1$, a tumor suppressor and negative regulator of $R A S$ in cutaneous melanoma (Figure 3). ${ }^{27} \mathrm{NF} 1$ mutations were present in 3 of 15 cases $(20 \%)$, thereby representing the second most frequent recurrent single-gene mutation in our series, after KIT mutations (Figure 2,Table 2). We identified one frameshift in NF1 in one case, and two tumors carried two NF1 variants each, all of which constitute putative loss-of-function mutations (Figure 3). ${ }^{28,29}$ As expected, oncogenic mutations in $K I T, R A S$ isoforms and BRAF were mutually exclusive (Figure 2). Furthermore, these mutations were also mutually exclusive with NF1 mutations, indicating significance of NF1 loss as a newly identified oncogenic event in anorectal melanoma.

\section{Recurrent SF3B1 Mutations in Anorectal Melanoma}

Three cases showed mutations in SF3B1, previously described in uveal melanoma and at very low frequency in cutaneous melanoma, but not, to our knowledge, in mucosal melanoma ${ }^{30,31}$ (Figure $4 a$ ). In all three cases, mutations occurred at codon 625, located in the fifth HEAT (Huntingtin, elongation factor 3, protein phosphatase 2A subunit PR65/A, TOR1) domain repeat, ${ }^{32}$ and comprised one R625H and two R625C substitutions (Table 2). An overwhelming majority of SF3B1 mutations in uveal melanomas occur at this residue (Figure 4a), and our findings identify SF3B1 (R625) mutations as a recurrent event also in mucosal melanomas. Interestingly, codon 625 mutations do not predominate in hematological malignancies, as mutations in codons $622,662,666$, and 700 are frequent in myelodysplastic syndrome as well as chronic lymphocytic leukemia/small lymphocytic leukemia (http://can cer.sanger.ac.uk/cosmic, Figure 4b). In our series of anorectal melanomas, SF3B1 (R625) mutations were seen in combination with other driver events, as two tumors carried activating KIT mutations and one tumor showed a deleterious mutation in NF1 (Figure 2).

\section{A subset of Anorectal Melanomas Carry Mutations in Genes Affecting Genomic Stability}

Five cases in our series demonstrated mutations in genes involved in DNA damage repair (TP53, BRCA1, and MLH1). Missense mutations in TP53 
NF1
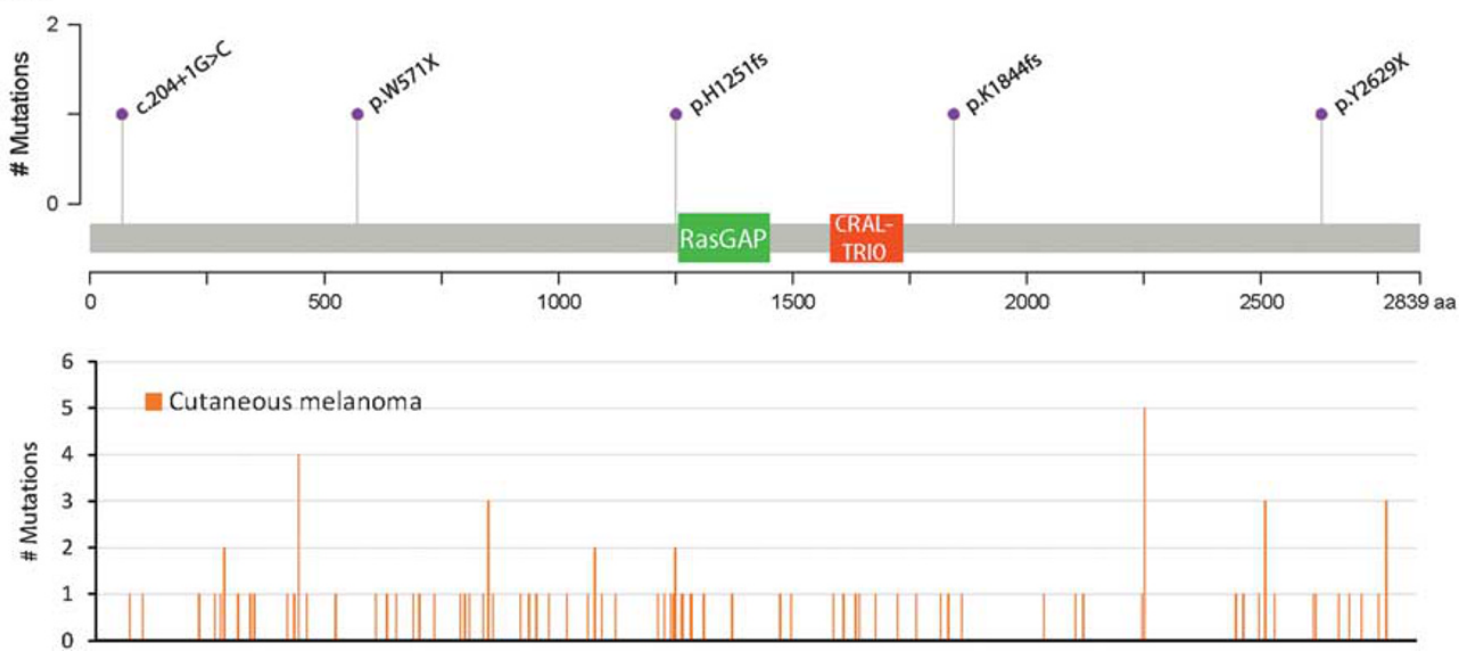

Figure 3 NF1 mutations in anorectal melanoma, schematic representation (top). Mutations are distributed throughout the protein. RasGAP: GTPase-activator protein for Ras-like GTPase domain (AA 1256-1451); CRAL-TRIO, C-terminal CRAL-TRIO phospholipid binding domain (AA 1591-1736). Frequency of NF1 mutations in cutaneous melanoma, as reported in the COSMIC database (bottom).

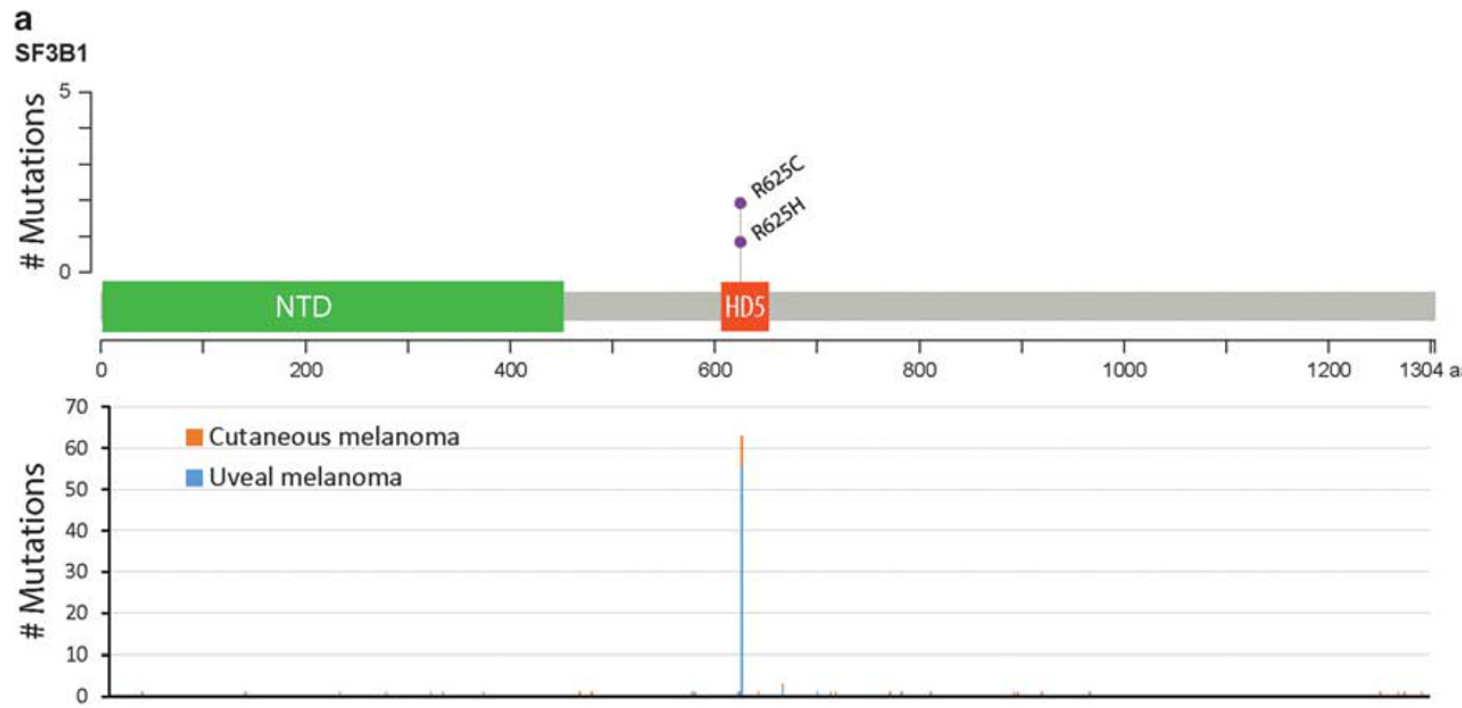

b

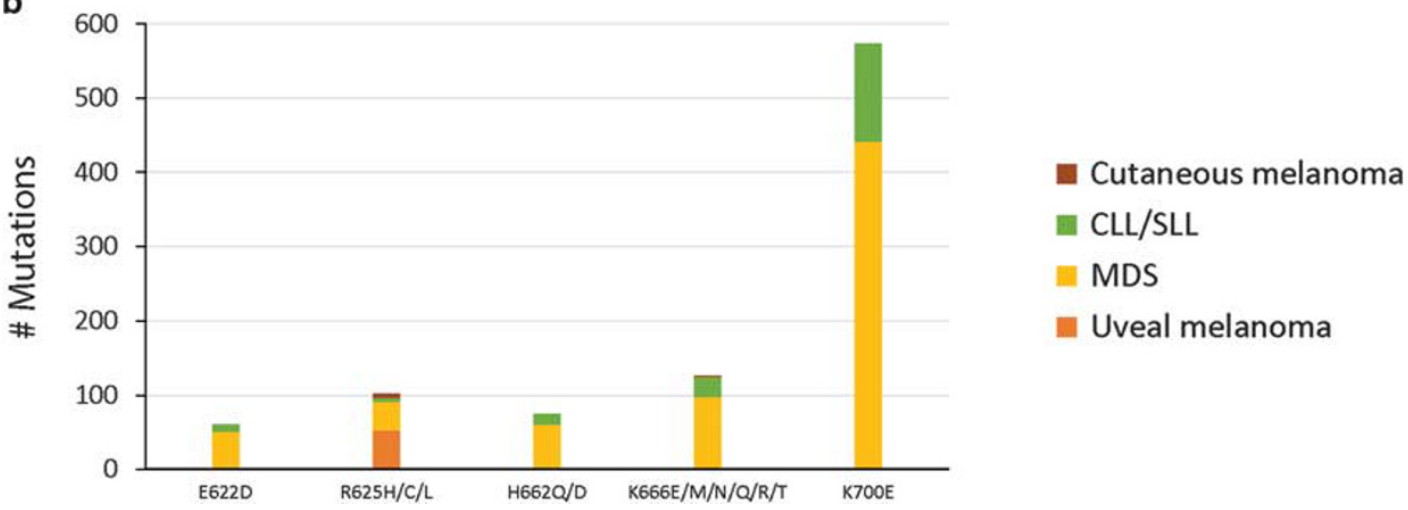

Figure 4 Distribution of SF3B1 mutations. (a) SF3B1 mutations in anorectal melanoma, schematic representation (top). Recurrent mutations are clustered in the region encoding HEAT domain 5. HD, HEAT domain; NTD, N-terminal domain (AA 1-450). Frequency of SF3B1 mutations in cutaneous and uveal melanomas, as reported in the COSMIC database (bottom). (b) Frequency of SF3B1 hotspot mutations by the major tumor types harboring these variants, as reported in the COSMIC database. CLL/SLL, chronic lymphocytic leukemia/small lymphocytic leukemia; MDS, myelodysplastic syndrome. 
a
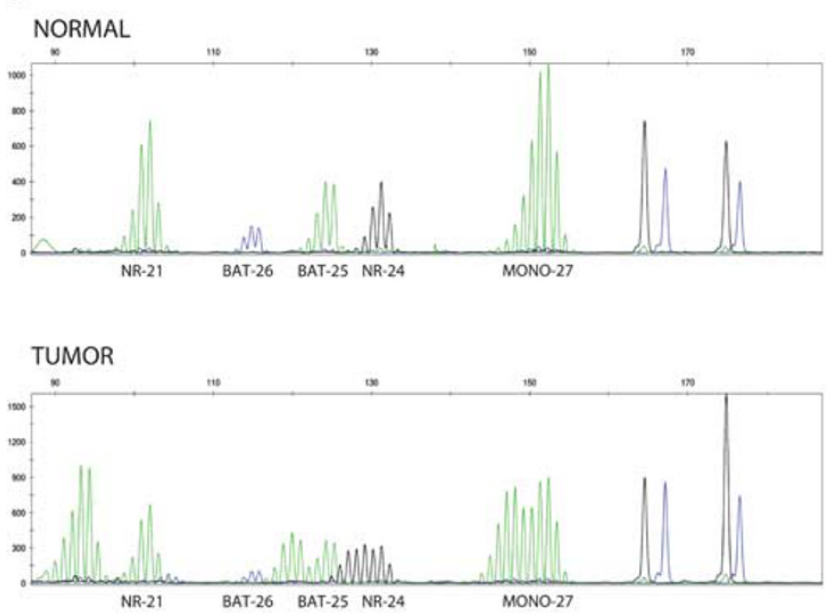

b
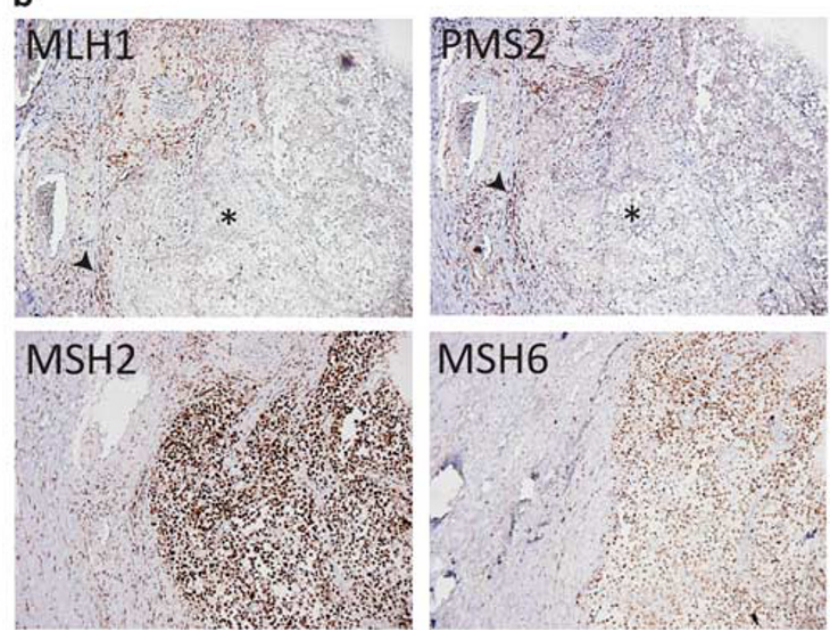

Figure 5 Evidence of genetic instability in anorectal melanoma with MLH1 (G67R) mutation (case 7). (a) Microsatellite instability testing by PCR shows altered alleles at $5 / 5$ tested loci in the tumor (bottom panel), but not in normal control tissue (top panel). (b) Immunohistochemistry. Loss of nuclear expression of MLH1 and PMS2 in melanoma cells (asterisk). Expression of MSH2 and MSH6 is retained. Note retained expression of all mismatch repair proteins in peripheral stromal tissue (arrowhead) (original magnification $\times 100$ ).

were found in three of 15 cases (20\%), two of which were previously reported as pathogenic mutations involving the DNA binding domain (Y220C, C242Y), as well as one nonsense mutation involving the N-terminal/transactivation domain (Table 2). Furthermore, whereas one tumor showed a deleterious frameshift mutation in BRCA1 (T557fs), a tumor suppressor gene involved in DNA repair and homologous recombination, in one case we detected a pathogenic MLH1 (G67R) mutation, reported previously in individuals with hereditary non-polyposis colorectal cancer syndrome (Lynch syndrome). ${ }^{33}$ The variant allele frequency was $53 \%$, suggesting a heterozygous MLH1 mutation (Supplementary Table 1). This case showed a higher mutation rate (33 variants) compared to the average number (14.1) of variants detected in our panel of 467 genes tested, and furthermore showed multiple frameshift mutations at mononucleotide repeat sequences involving additional cancer-related genes, consistent with a mutator phenotype (Figure 2, Supplementary Table 1). PCR testing in this tumor confirmed MSI in five of five loci ('MSI-High', Figure 5a), whereas immunohistochemical analysis for mismatch repair proteins MLH1, MSH2, PMS2, and MSH6 demonstrated loss of MLH1 protein expression, as well as loss of dimerization partner PMS2, as expected ${ }^{34}$ (Figure 5b). Interestingly, although this patient was elderly (81y) with a reported history of prostate cancer, clinical features were not typical of Lynch syndrome, as a personal and family history of colon cancer was lacking.

\section{Discussion}

Melanoma is a heterogeneous disease, characterized by distinct molecular subsets based on anatomic site as well as level of sun-exposure. ${ }^{35}$ Here, we describe the mutational landscape in a series of 15 anorectal melanomas, a rare melanoma subtype with an annual incidence of $<1.0$ per million population in the United States, which nevertheless shows a rising incidence. ${ }^{1}$ Importantly, we identify driver events in 14 of 15 anorectal melanomas, a majority of which representing potentially actionable mutations.

Primary oncogenic events previously described in melanomas arising in the oral mucosa and anogenital region include activating KIT mutations in $15-30 \%$ of cases, ${ }^{11-13}$ whereas oncogenic $B R A F$ mutations are infrequent $(\sim 6 \%) .{ }^{36}$ In a large case series analyzing 26 anorectal melanomas for $B R A F$, NRAS, KIT, and PDGFRA mutations, KIT mutations were detected in three cases (15\%), one tumor (5\%) harbored an NRAS mutation and BRAF point mutations were absent. ${ }^{11}$ Our findings confirm recurrent activating KIT mutations in anorectal melanomas, which represented the predominant single-gene mutation in our series $(5 / 15$ cases, $33 \%$ ). Whereas three of five KIT mutations affected the juxtamembrane domain (exon 11) and are expected to predict response to c-Kit inhibitors, ${ }^{15,17,37}$ imatinib may be less effective in melanomas with mutations involving the distal kinase domain. ${ }^{16} \mathrm{~A}$ partial response to imatinib was reported in one patient with metastatic mucosal melanoma with a KIT exon 17 mutation (D820Y, present in one case of our anorectal melanoma series), ${ }^{17}$ but disease progression observed in another patient with metastatic acral melanoma harboring the same mutation. ${ }^{15}$ In addition to imatinib, multikinase inhibitor sorafenib and newer-generation tyrosine kinase inhibitors such as nilotinib, dasatinib, and masitinib have shown some efficacy in metastatic mucosal melanomas harboring KIT exon 11 or 13 mutations. ${ }^{38,39}$ However, development of resistance to c-Kit 
inhibition is common, ${ }^{40}$ and although nilotinib may achieve temporary disease control in some imatinibresistant mucosal melanomas, ${ }^{18}$ these examples illustrate limitations for c-Kit inhibitor-based therapy.

We did not assess copy number variation in the present study, and KIT amplifications, which can occur in KIT-mutant as well as KIT-wt tumors, may represent an additional mechanism of elevated c-Kit activity in our series of anorectal melanomas. However, mucosal melanomas harboring only KIT amplifications alone were found to be largely insensitive to tyrosine kinase inhibitors. ${ }^{17,37}$

Mutation frequency in RAS and its canonical downstream effectors was low in our series as expected, and interestingly, revealed variants that are rarely observed in cutaneous melanomas: HRAS hotspot mutations, present in one case of anorectal melanoma, occur in $10-15 \%$ of Spitz nevi but in $<1 \%$ of cutaneous melanomas. ${ }^{25,41}$ Furthermore, we identified a duplication of threonine in the $B R A F$ activation loop (p.T599dup), a rare mutation with only one case reported in melanoma, ${ }^{42,43}$ and previously not described in mucosal melanomas. Interestingly, BRAF (T599dup) mutations are also seen in rare cases of colorectal adenocarcinomas, ${ }^{44}$ and display in vitro kinase activity and cellular MEK/ERK activation potential comparable to $B R A F$ (V600E). ${ }^{26}$ Of note, BRAF (T599dup) is not detected by immunohistochemistry using BRAF-antibody VE1, ${ }^{45}$ and our findings therefore demonstrate that more extensive sequencing is warranted in anorectal melanomas negative for NRAS/BRAF hotspot mutations. Consistent with previous case reports in mucosal melanomas, activating mutations in KIT, $B R A F$, and $R A S$ isoforms were mutually exclusive in our series of anorectal melanomas.

To our knowledge, this is the first study to identify NF1 loss-of-function mutations as a recurrent mutational event in melanomas of the anal canal. NF1 mutations showing $\mathrm{C}>\mathrm{T}$ transitions are known to occur in cutaneous melanomas lacking BRAF/ NRAS hotspot mutations, and are also prevalent in pure desmoplastic melanomas, implicating UVradiation as an oncogenic factor in these melanoma subtypes. ${ }^{28,29,41,46}$ However, our findings of NF1 mutations in $20 \%$ of anorectal melanomas in which sunlight as an etiologic factor can be excluded suggest alternative mechanisms of mutagenesis. As a RAS-specific GTPase-activating protein, NF1 negatively regulates RAS and thereby MAPK pathway activity. Importantly, NF1 loss-of-function was associated with RAS activation as well as with MEK dependence in melanoma cell lines, although other studies indicated that NF1 suppression is not always associated with MEK-inhibitor response in vitro. ${ }^{27,47}$ Nevertheless, MEK inhibition is an attractive strategy for tumors with NF1 loss-of-function mutations, ${ }^{38}$ and phase II clinical trials for Selumetinib-based therapy of inoperable neurofibromas in patients with germline NF1 loss-of-function mutations are currently underway (clinicaltrials.gov). As a group, we identified oncogenic events leading to MAPK pathway hyperactivation in 6 of 15 cases (40\%), either by rare activating mutations in $B R A F$ or $R A S$ isoforms, or by NF1 loss-of-function mutations, implicating opportunities for MEK inhibitor-based therapy in a significant subset of anorectal melanomas.

Interestingly, three cases in our series demonstrated mutations in SF3B1 at codon 625. In uveal melanoma, $S F 3 B 1$ mutations occur exclusively at codon 625 and correlate with absence of adverse prognostic factors, such as monosomy 3 and mutations in $B A P 1 .{ }^{30}$ In cutaneous melanoma, SF3B1 (R625) mutations occur at very low frequency $(<1 \%),{ }^{31}$ and to our knowledge our study is the first to identify recurrent SF3B1 codon 625 mutations in mucosal melanomas (20\%). Interestingly, SF3B1 mutation pattern and correlation with outcome appear to be tumor-specific: mutations at codon 700 predominate in hematological malignancies as well as in breast cancer, and are associated with favorable outcome in myelodysplastic syndrome, but with poor survival in chronic lymphocytic leukemia. ${ }^{48}$ Functionally, loss of $S F 3 B 1$, which encodes subunit 1 of the RNA splicing factor $3 \mathrm{~b}$ protein complex, leads to missplicing of critical neural crest transcription factors in a zebrafish model. ${ }^{49}$ SF3B1 mutations identified in uveal melanoma, however, do not appear to be associated with missplicing, and their functional consequences are unknown. ${ }^{30}$ Whether SF3B1 (R625) mutations are prognostically significant in mucosal melanoma remains to be determined-in our series, all three patients whose tumors carried SF3B1 mutations developed visceral or nodal metastases. Although our case numbers are limited, these findings contrast with previous studies showing that SF3B1 (R625) mutations are rare in metastatic uveal melanoma. ${ }^{30}$

Similar to findings in cutaneous melanoma, ${ }^{29,41}$ mutations in tumor suppressor genes $N F 1$ and BRCA1 in our series of anorectal melanomas occurred in tumors lacking hotspot mutations in RAS or KIT. However, two tumors showed concurrent mutations in KIT and TP53. TP53 mutations have been reported in up to $19 \%$ of cutaneous melanomas overall, with increased mutation frequencies detected in desmoplastic melanomas and melanomas occurring on chronically sun-damaged skin. ${ }^{28,29,50}$ Interestingly, a previous study identified non-silent TP53 mutations in $28 \%$ of mucosal melanomas, including in three anal melanomas. ${ }^{51}$ Similarly, TP53 mutations were observed at a frequency of $18 \%$ in a recent study of melanomas of the female genital tract. ${ }^{52}$ Our study therefore confirms TP53 mutations as a recurrent mutational event in anorectal melanomas $(20 \%)$. We furthermore identified pathogenic mutations in one case each for BRCA1 and MLH1. Although carriers of BRCA2 germline mutations have an increased risk for development of cutaneous melanoma, ${ }^{53}$ 
associations with BRCA1 mutations are less well established, and to our knowledge the deleterious BRCA1 (T557fs) mutation described here has not been previously identified in melanoma tissue.

The pathogenic MLH1 (G67R) mutation identified in our anorectal melanoma series was previously reported in several Lynch syndrome families, and deleteriously affects protein function. ${ }^{33,54}$ Accordingly, this tumor demonstrated MSI and loss of mismatch repair protein expression, and furthermore showed the highest mutational load of all cases. Interestingly, this tumor carried several frameshift mutations at mononucleotide repeat sequences involving additional cancer-related genes, suggesting that these mutational events occurred secondary to genomic instability. As allele frequency estimates suggested a heterozygous MLH1 mutation (variant allele frequency $53 \%$ ), inactivation of the second allele in this case was likely epigenetic. Notably, the clinical history did not support a diagnosis of Lynch syndrome in this elderly patient (81 years of age), making an MLH1 germline mutation unlikely. These findings furthermore contrast with the low frequency of mismatch repair gene mutations in sporadic MSIHigh colon cancers. ${ }^{55}$ In cutaneous melanoma, lack of comprehensive mutation analysis in the majority of studies to date precludes distinction between epigenetic inactivation versus primary mutational events. ${ }^{56}$ Biallelic somatic inactivation of $M L H 1$ through chromosomal deletion and splice site mutation, respectively, has been described in one case of primary cutaneous melanoma, and deletions of entire exons of the MLH1 gene occur in a significant proportion of cutaneous melanomas and correlate with decreased patient survival. ${ }^{57,58}$ Taken together, our findings implicate MSI in the pathogenesis of a subset of anorectal melanomas, and together with the identification of deleterious $B R C A 1$ as well as TP53 mutations are in agreement with the hypothesis that genomic instability has a role in tumor development. ${ }^{59}$

Finally, we note that activating mutations in GNAQ/GNA11-seen in blue nevi and related melanocytic neoplasms, as well as in uveal melanomas ${ }^{60}$ -are absent in anorectal melanomas, in accordance with studies reporting absence of GNAQ/GNA11 mutations in melanomas of the female genital tract. ${ }^{52,61}$ No mutations were furthermore identified in beta-catenin, a gene infrequently mutated in socalled 'triple-wild type' cutaneous melanomas, which lack BRAF, NRAS, and NF1 mutations. ${ }^{41}$

In summary, whereas previous reports describe driver events such as activating KIT mutations in up to $30 \%$ of mucosal melanomas, our study reveals that comprehensive molecular analysis could identify melanoma-associated mutational events in the majority of anorectal melanomas. Furthermore, we show that anorectal melanomas are genetically heterogeneous. Predominant molecular subgroups include KIT-mutant tumors (33\%), as well as tumors carrying mutations expected to result in MAPK pathway hyperactivation (40\%). The latter group includes recurrent loss-of-function mutations in NF1 ( $20 \%$ of cases), a novel finding in mucosal melanomas, as well as rare mutations targeting $B R A F$ and $R A S$ isoforms, and extended sequence analysis in anorectal melanomas negative for BRAF/NRAS hotspot mutations is warranted. Importantly, as these mutations as well as NF1 mutations appear to be mutually exclusive with KIT mutations in anorectal melanoma, our findings raise the possibility that MEKi may expand the armamentarium of effective targeted therapeutics for patients whose tumors lack actionable KIT mutations. In this regard, although evidence for efficacy of MEKi in BRAF/NRAS-wt melanomas is still sparse, patients with rare tumors such as anorectal melanoma may benefit from novel clinical trial designs ('basket' studies), which aim to exploit putative predictive biomarkers to increase eligibility for patients with rare heterogeneous diseases, rather than focusing on cancer entities. ${ }^{62}$ A third molecular group identified in our series of anorectal melanomas represents tumors with a spectrum of mutations potentially affecting genomic stability (BRCA1, MLH1, TP53). The significance of recurrent SF3B1 (R625) mutations in mucosal melanoma, also a novel finding of this study, remains to be determined.

\section{Acknowledgments}

We are grateful to technical support staff of the Personalized Genomic Medicine Laboratory, Columbia University for tumor microdissection.

\section{Disclosure/conflict of interest}

The authors declare no conflict of interest.

\section{References}

1 Chen $\mathrm{H}$, Cai Y, Liu Y, et al. Incidence, surgical treatment, and prognosis of anorectal melanoma from 1973 to 2011: a population-based SEER analysis. Medicine (Baltimore) 2016;95:e2770.

2 Carvajal RD, Spencer SA, Lydiatt W. Mucosal melanoma: a clinically and biologically unique disease entity. J Natl Compr Canc Netw 2012;10:345-356.

3 Cote TR, Sobin LH. Primary melanomas of the esophagus and anorectum: epidemiologic comparison with melanoma of the skin. Melanoma Res 2009;19: 58-60.

4 Ragnarsson-Olding BK, Nilsson PJ, Olding LB, et al. Primary ano-rectal malignant melanomas within a population-based national patient series in Sweden during 40 years. Acta Oncol 2009;48:125-131.

5 Fenger C, Lyon H. Endocrine cells and melanincontaining cells in the anal canal epithelium. Histochem J 1982;14:631-639.

6 Clemmensen OJ, Fenger C. Melanocytes in the anal canal epithelium. Histopathology 1991;18:237-241. 
7 Werdin C, Limas C, Knodell RG. Primary malignant melanoma of the rectum. Evidence for origination from rectal mucosal melanocytes. Cancer 1988;61: 1364-1370.

8 Ballantyne AJ. Malignant melanoma of the skin of the head and neck. An analysis of 405 cases. Am J Surg 1970;120:425-431.

9 Iddings DM, Fleisig AJ, Chen SL et al. Practice patterns and outcomes for anorectal melanoma in the USA, reviewing three decades of treatment: is more extensive surgical resection beneficial in all patients? Ann Surg Oncol 2010;17:40-44.

10 Tacastacas JD, Bray J, Cohen YK, et al. Update on primary mucosal melanoma. J Am Acad Dermatol 2014;71:366-375.

11 Antonescu CR, Busam KJ, Francone TD, et al. L576P KIT mutation in anal melanomas correlates with KIT protein expression and is sensitive to specific kinase inhibition. Int J Cancer 2007;121:257-264.

12 Curtin JA, Busam K, Pinkel D, et al. Somatic activation of KIT in distinct subtypes of melanoma. J Clin Oncol 2006;24:4340-4346.

13 Santi R, Simi L, Fucci R, et al. KIT genetic alterations in anorectal melanomas. J Clin Pathol 2015;68: 130-134.

14 Mihajlovic M, Vlajkovic S, Jovanovic P, et al. Primary mucosal melanomas: a comprehensive review. Int J Clin Exp Pathol 2012;5:739-753.

15 Carvajal RD, Antonescu CR, Wolchok JD, et al. KIT as a therapeutic target in metastatic melanoma. JAMA 2011;305:2327-2334.

16 Guo J, Si L, Kong Y, et al. Phase II, open-label, singlearm trial of imatinib mesylate in patients with metastatic melanoma harboring c-Kit mutation or amplification. J Clin Oncol 2011;29:2904-2909.

17 Hodi FS, Corless CL, Giobbie-Hurder, et al. Imatinib for melanomas harboring mutationally activated or amplified KIT arising on mucosal, acral, and chronically sundamaged skin. J Clin Oncol 2013;31:3182-3190.

18 Carvajal RD, Lawrence DP, Weber JS, et al. Phase II study of nilotinib in melanoma harboring KIT alterations following progression to prior KIT inhibition. Clin Cancer Res 2015;21:2289-2296.

19 Martinez-Cadenas C, Bosch N, Penas L, et al. Malignant melanoma arising from a perianal fistula and harbouring a BRAF gene mutation: a case report. BMC Cancer 2011;11:343.

$20 \mathrm{Ni} \mathrm{S}$, Huang D, Chen X, et al. c-kit gene mutation and CD117 expression in human anorectal melanomas. Hum Pathol 2012;43:801-807.

21 Satzger I, Schaefer T, Kuettler U, et al. Analysis of c-KIT expression and KIT gene mutation in human mucosal melanomas. Br J Cancer 2008;99:2065-2069.

22 Ganapathi KA, Jobanputra V, Iwamoto F, et al. The genetic landscape of dural marginal zone lymphomas. Oncotarget 2016; doi:10.18632/oncotarget.9678 (e-pub ahead of print 27 May 2016)..

23 Horst BA, Terrano D, Fang Y, et al. 9p21 gene locus in Spitz nevi of older individuals: absence of cytogenetic and immunohistochemical findings associated with malignancy. Hum Pathol 2013;44:2822-2828.

24 Stratton MR, Campbell PJ, Futreal PA. The cancer genome. Nature 2009;458:719-724.

25 Bastian BC, LeBoit PE, Pinkel D. Mutations and copy number increase of HRAS in Spitz nevi with distinctive histopathological features. Am J Pathol 2000;157: 967-972.
26 Eisenhardt AE, Olbrich H, Roring M, et al. Functional characterization of a BRAF insertion mutant associated with pilocytic astrocytoma. Int J Cancer 2011;129: 2297-2303.

27 Nissan MH, Pratilas CA, Jones AM, et al. Loss of NF1 in cutaneous melanoma is associated with RAS activation and MEK dependence. Cancer Res 2014;74:2340-2350.

28 Hodis E, Watson IR, Kryukov GV, et al. A landscape of driver mutations in melanoma. Cell 2012;150:251-263.

29 Krauthammer $\mathrm{M}$, Kong $\mathrm{Y}$, Ha BH, et al. Exome sequencing identifies recurrent somatic RAC1 mutations in melanoma. Nat Genet 2012;44:1006-1014.

30 Harbour JW, Roberson ED, Anbunathan $\mathrm{H}$, et al. Recurrent mutations at codon 625 of the splicing factor SF3B1 in uveal melanoma. Nat Genet 2013;45: 133-135.

31 Kong Y, Krauthammer M, Halaban R. Rare SF3B1 R625 mutations in cutaneous melanoma. Melanoma Res 2014;24:332-334.

32 Andrade MA, Bork P. HEAT repeats in the Huntington's disease protein. Nat Genet 1995;11:115-116.

33 Peltomaki P, Vasen HF. Mutations predisposing to hereditary nonpolyposis colorectal cancer: database and results of a collaborative study. The International Collaborative Group on Hereditary Nonpolyposis Colorectal Cancer. Gastroenterology 1997;113: 1146-1158.

34 Hall G, Clarkson A, Shi, et al. Immunohistochemistry for PMS2 and MSH6 alone can replace a four antibody panel for mismatch repair deficiency screening in colorectal adenocarcinoma. Pathology 2010;42: 409-413.

35 Curtin JA, Fridlyand J, Kageshita T, et al. Distinct sets of genetic alterations in melanoma. $\mathrm{N}$ Engl J Med 2005;353:2135-2147.

36 Hocker T, Tsao H. Ultraviolet radiation and melanoma: a systematic review and analysis of reported sequence variants. Hum Mutat 2007;28:578-588.

37 Minor DR, Kashani-Sabet M, Garrido M, et al. Sunitinib therapy for melanoma patients with KIT mutations. Clin Cancer Res 2012;18:1457-1463.

38 Johnpulle RA, Johnson DB, Sosman JA. Molecular targeted therapy approaches for BRAF wild-type melanoma. Curr Oncol Rep 2016;18:6.

39 Prosvicova J, Lukesova S, Kopecky J, et al. Rapid and clinically significant response to masitinib in the treatment of mucosal primary esophageal melanoma with somatic KIT exon 11 mutation involving brain metastases: a case report. Biomed Pap Med Fac Univ Palacky Olomouc Czech Repub 2015;159:695-697.

40 Bello DM, Dematteo RP, Ariyan CE. The GIST of targeted therapy for malignant melanoma. Ann Surg Oncol 2014;21:2059-2067.

41 Cancer Genome Atlas N. Genomic classification of cutaneous melanoma. Cell 2015;161:1681-1696.

42 Long GV, Menzies AM, Nagrial AM, et al. Prognostic and clinicopathologic associations of oncogenic BRAF in metastatic melanoma. J Clin Oncol 2011;29: 1239-1246.

43 Menzies AM, Haydu LE, Visintin L, et al. Distinguishing clinicopathologic features of patients with V600E and V600K BRAF-mutant metastatic melanoma. Clin Cancer Res 2012;18:3242-3249.

44 Mostert B, Jiang Y, Sieuwerts AM, et al. KRAS and BRAF mutation status in circulating colorectal tumor cells and their correlation with primary and metastatic tumor tissue. Int J Cancer 2013;133:130-141. 
45 Long GV, Wilmott JS, Capper D, et al. Immunohistochemistry is highly sensitive and specific for the detection of V600E BRAF mutation in melanoma. Am J Surg Pathol 2013;37:61-65.

46 Wiesner T, Kiuru M, Scott SN, et al. NF1 mutations are common in desmoplastic melanoma. Am J Surg Pathol 2015;39:1357-1362.

47 Krauthammer M, Kong Y, Bacchiocchi et al. Exome sequencing identifies recurrent mutations in NF1 and RASopathy genes in sun-exposed melanomas. Nat Genet 2015;47:996-1002.

48 Papaemmanuil E, Cazzola M, Boultwood J, et al. Somatic SF3B1 mutation in myelodysplasia with ring sideroblasts. N Engl J Med 2011;365:1384-1395.

49 An M, Henion PD. The zebrafish sf3b1b460 mutant reveals differential requirements for the sf3b1 premRNA processing gene during neural crest development. Int J Dev Biol 2012;56:223-237.

50 Shain AH, Garrido M, Botton T, et al. Exome sequencing of desmoplastic melanoma identifies recurrent NFKBIE promoter mutations and diverse activating mutations in the MAPK pathway. Nat Genet 2015;47: 1194-1199.

51 Ragnarsson-Olding BK, Karsberg S, Platz, et al. Mutations in the TP53 gene in human malignant melanomas derived from sun-exposed skin and unexposed mucosal membranes. Melanoma Res 2002;12: 453-463.

52 Yelamos O, Merkel EA, Sholl LM, et al. Nonoverlapping clinical and mutational patterns in melanomas from the female genital tract and atypical genital nevi. J Invest Dermatol 2016;136:1858-1865.

53 Breast Cancer Linkage C. Cancer risks in BRCA2 mutation carriers. J Natl Cancer Inst 1999;91:1310-1316.
54 Avdievich E, Reiss C, Scherer SJ, et al. Distinct effects of the recurrent Mlh1G67R mutation on MMR functions, cancer, and meiosis. Proc Natl Acad Sci USA 2008;105:4247-4252.

55 Kuismanen SA, Holmberg MT, Salovaara R, et al. Genetic and epigenetic modification of MLH1 accounts for a major share of microsatellite-unstable colorectal cancers. Am J Pathol 2000;156:1773-1779.

56 Richman S. Deficient mismatch repair: read all about it (Review). Int J Oncol 2015;47:1189-1202.

57 Castiglia D, Pagani E, Alvino E, et al. Biallelic somatic inactivation of the mismatch repair gene MLH1 in a primary skin melanoma. Genes Chromosomes Cancer 2003;37:165-175.

58 Korabiowska M, Brinck U, Stachura J, et al. Exonic deletions of mismatch repair genes MLH1 and MSH2 correlate with prognosis and protein expression levels in malignant melanomas. Anticancer Res 2006;26: 1231-1235.

59 Whiteman DC, Pavan WJ, Bastian BC. The melanomas: a synthesis of epidemiological, clinical, histopathological, genetic, and biological aspects, supporting distinct subtypes, causal pathways, and cells of origin. Pigment Cell Melanoma Res 2011;24:879-897.

60 Van Raamsdonk CD, Bezrookove V, Green G, et al. Frequent somatic mutations of GNAQ in uveal melanoma and blue naevi. Nature 2009;457:599-602.

61 Tseng D, Kim J, Warrick, et al. Oncogenic mutations in melanomas and benign melanocytic nevi of the female genital tract. J Am Acad Dermatol 2014;71: 229-236.

62 Hyman DM, Puzanov I, Subbiah V, et al. Vemurafenib in multiple nonmelanoma cancers with BRAF V600 mutations. N Engl J Med 2015;373:726-736.

Supplementary Information accompanies the paper on Modern Pathology website (http://www.nature.com/ modpathol) 